\section{Research Square}

Preprints are preliminary reports that have not undergone peer review.

They should not be considered conclusive, used to inform clinical practice, or referenced by the media as validated information.

\title{
Evaluation of PRKAA2 Genetic Variation on Metformin Efficacy as an Initial Therapy Among Drug-Naïve Patients With Type II Diabetes Mellitus
}

Dita Virginia

Department of Pharmacology and Therapy, Faculty of Medicine, Public Health, and Nursing, Universitas Gadjah Mada

Mae Wahyuningsih

Department of Pharmacology and Therapy, Faculty of Medicine, Public Health, and Nursing, Universitas Gadjah Mada

Dwi Nugrahaningsih ( $\nabla$ dwi.aris.a@ugm.ac.id)

Department of Pharmacology and Therapy, Faculty of Medicine, Public Health, and Nursing, Universitas Gadjah Mada

\section{Research Article}

Keywords: PRKAA2, metformin, type 2 diabetes mellitus, genetic variation

Posted Date: November 24th, 2020

DOI: https://doi.org/10.21203/rs.3.rs-107043/v1

License: (c) This work is licensed under a Creative Commons Attribution 4.0 International License. Read Full License 


\section{Abstract}

Background: Metformin is the most popular oral antidiabetic agent, which is recommended as initial monotherapy. AMPK is the pivotal target of metformin molecular mechanisms. AMPK subunit a2 (encoded by PRKAA2) is a gene contributable to increase type 2 diabetes mellitus (T2DM) risk. This study aimed to evaluate PRKAA2 rs2796498, rs2746342, and rs980799 genetic variations on metformin efficacy.

Methods: This study enrolled 191 newly diagnosed Indonesia T2DM patients in primary health care. Patients who received metformin as monotherapy for at least 3 months were included for genotyping. Genotyping was performed using the Taqman assay.

Results: Baseline characteristics showed that BMI was higher among AA than $G G+A G(p=0.04)$. Patients with TT genotype showed a higher FBG and $H b A 1 c$ than $G G+G T$ ( $p=0.02$ and $p=0.02$, respectively). There was no significant difference in allele and genotype frequencies between responders and nonresponders group in PRKAA2 rs2796498, rs9803799, and rs2746342. However, among PRKAA2 rs2796498, AG carrier had 0.32 times of responding in metformin efficacy after adjusting BMI, WC, blood pressure, lipid profiles, and eGFR. Dominant model of rs2796498 showed a significant association $(\mathrm{OR}=0.34,95 \% \mathrm{Cl}=0.13-0.90)$ to metformin efficacy.

Conclusions: Our findings suggest that PRKAA2 rs2796498 genetic variation may affect metformin efficacy, especially AG carrier, in drug-naïve T2DM patients.

\section{Introduction}

Metformin is notable as the most prescribed medicine for T2DM patients as a recommended initial type 2 diabetes mellitus (T2DM) therapy by many international guidelines. Drug-naïve T2DM patients who have HbA1c 7-9\% are suggested to consume metformin until it reaches the maximum doses before adding a second agent [1, 2]. Metformin considerably has better safety and efficacy as initial T2DM therapy [3]. Metformin is a guanidine derivate which acts as an unchanged form. Metformin specifically reduces blood glucose by enhancing glucose uptake in peripheral tissue and suppressing glucose hepatic production [4-6]. The underlying complex molecular mechanism of metformin has been observed in many studies, and AMP-activated protein kinase (AMPK) was mentioned to have a pivotal role in metformin action [7, 8].

AMPK is a cellular energy sensor whose phosphorylation is stimulated by lower ATP concentration in cells. AMPK has three subunits consisting of a, b, and g. AMPK suppresses the anabolic process, which utilizes ATP [9]. Metformin stimulates phosphorylation of Thr-172 at a catalytic unit [10]. Some studies also demonstrated that metformin activates AMPK after inhibiting mitochondrial complex I respiratory chain. Thus it reduces the ATP level [8, 11]. Furthermore, other studies reported that LKB-1 is also involved in AMPK activation by metformin [12]. Besides, AMPK via acetyl-CoA carboxylase 1 (ACC1) and ACC2 could inhibit malonyl CoA synthesis. As a result, it reduces lipogenesis and increases insulin sensitivity [13]. Therefore, metformin does not affect weight gain.

Nevertheless, there is a different efficacy of metformin among T2DM patients [14]. Metformin efficacy is affected by various factors, including age, lifestyle, baseline $\mathrm{HbA} 1 \mathrm{c}$, adherence [15, 16], gene-drug interaction, and gene-environment interaction [17]. Inter-individual variability plays an important role in drug response affected by drug-gene interaction. Therefore, pharmacogenomic still be a promising study for discovering the relationship between gene and medicine's pharmacodynamic and pharmacokinetic, in this context especially metformin efficacy.

Many studies have explored the effect of genetic variation on metformin response in the pharmacokinetic area [18, 19]. Otherwise, a little study conduct pharmacogenomic research focused on the pharmacodynamic of metformin. Accordingly, it is relevant to explore pharmacogenetic concerning the pharmacodynamic of metformin in achieving the HbA1c goal. Since AMPK is involved as the main target in the metformin molecular mechanism, genes coding AMPK may have contributable to metformin efficacy. A recent review declared that PRKAA2 coding AMPKa2 is one of a gene related to clinical outcomes after metformin therapy [20]. Nevertheless, that review has not mentioned an SNP of PRKAA2 contributing to metformin response yet.

Previous studies have reported an association of PRKAA2 with susceptibility of T2DM (rs2796498 and rs2746342) [21, 22], even in metformin effectiveness (rs9803799) [23]. However, no studies observe the association between PRKAA2 rs2796498 and rs2746342 genetic variation and the effectiveness of metformin use. Notably, those three SNP have not been explored yet in Indonesia. Therefore, this study aimed to observe the genotype frequency among T2DM Indonesian patients receiving metformin. Furthermore, this study investigates the influence of PRKAA2 rs2796498, rs9803799, and rs2746342 genetic variation on the efficacy of metformin in drug-naïve T2DM patients, determined by the reduction of HbA1c level.

\section{Methods}

\section{Participants}

This study recruited 191 participants from ten primary health care (PHC) in Yogyakarta Province, Indonesia. Physicians in PHC gave the first prescription of metformin on newly diagnosed T2DM patients. Then participants were followed up for three months. The participant's selection following inclusion criteria: newly diagnosed T2DM patients with $\mathrm{HbA} 1 \mathrm{c} \geq 7 \%$, 18-70 years old, do not consume other hyperglycemia agents, either oral or injectable dosage forms. Participants were excluded when GFR $<30 \mathrm{ml} / \mathrm{min} / \mathrm{m} 2$, albumin serum $3.4-4.8 \mathrm{~g} / \mathrm{dL}$, and diagnosed as diabetes gestational. Finally, only $106 \mathrm{newly}$ diagnosed T2DM patients who had taken the first prescription metformin as monotherapy and completed follow up for three consecutive months were enrolled in this study. Patients were classified into two groups: metformin responders $(n=45)$ and metformin non-responders ( $n=61)$. All of our participants were of Indonesia origin. Metformin responders are patients who could achieve decreasing $\mathrm{HbA} 1 \mathrm{c}>1.12 \%$ during follow up [24]. 
Anthropometric measurement, blood pressure, blood sample collecting for clinical chemistry and genotyping analysis, and medical record data were obtained for this study after patients signing informed consent. All participants were briefed about the study aim, procedure, duration, potential risk, and benefit. The study was performed in compliance, according to the Declaration of Helsinki. The study protocol was approved by the Medical and Health Research Ethics Committee (MHREC) Faculty of Medicine, Public Health, and Nursing Universitas Gadjah Mada - Dr. Sardjito General Hospital. Patients had the right not to participate in our study at any time.

\section{Clinical measurement}

Our study collected demographic data, anthropometric data, and laboratory results. Age and gender, as demographic data and metformin dose, were obtained from medical records. Nutritionists measured anthropometric measurements, including body weight, height, and waist circumference. Body Mass Index (BMI) was calculated by divided body weight $(\mathrm{kg})$ by height $(\mathrm{m} 2)$. Blood pressure was measured by the nurse in early registration. We examined laboratory results, including FBG, HbA1c, creatinine serum, HDL-c, Triglyceride-c, and total cholesterol. Laboratory data were collected by a PHC analyst that helped by a commercial laboratory after an overnight fast. FBG and creatinine serum was measured by the hexokinase method and enzymatic method, respectively. $\mathrm{HbA} 1 \mathrm{c}$ was calculated using high-performance liquid chromatography (Cobas D-10). Glomerular filtration rate (GFR) was calculated using CKD-EPI formulation using the creatinine level. Lipid profiles, including HDL-c, Triglyceride-c, and total cholesterol, were measured using Cobas C311. LDL was calculated using Friedewald formulation.

DNA extraction and genotyping

According to the kit protocol, genomic DNA was extracted from peripheral whole blood-EDTA using Geneaid® Blood DNA Mini Kit and stored at -20 until the genotyping procedure. Genotyping in rs 2796498 , rs 9803799 , and rs 2746342 was performed using the TaqMan® genotyping assay and Applied Biosystems $\AA$ qPCR 7500 Fast Real-Time PCR System. The final reaction volume using in real-time PCR is $10 \mathrm{~mL}$, including $2.5 \mathrm{~mL}$ nucleotide-free water, $5 \mathrm{~mL}$ TaqMan GTXpress mix, $0.5 \mathrm{~mL}$ TaqMan SNP genotyping assay, and $2 \mathrm{~mL}$ of genomic DNA. The thermal cycle for a reaction was as follows: $40 \mathrm{cycles}$ at hold $95^{\circ} \mathrm{C}$ for the $20 \mathrm{~s}$, at denaturing $95^{\circ} \mathrm{C}$ for $3 \mathrm{~s}$, and then annealing $60^{\circ} \mathrm{C}$ for $30 \mathrm{~s}$. PRKAA2 SNPs were determined using the following primer sequences:

rs2796498: CTGTAACAGTGTTAGTGATTTAAAC[A_G]GAGAGAGCAACCTTACCCTTTCAGT

rs9803799: TAAATACAGGGTTTATATCCCCACA[G/T]TCAATGTAAATTCCTTTTTTTAAAA

rs2746342: AGAGAGGCTAAGATGCAGGCTGTAC[G/T]CTGGGTAGCCATGTACTCAGTTGTA

\section{Statistical analysis}

The baseline and follow-up participant's characteristics were analyzed and compared between the genotype model using an independent t-test or chi-square, as appropriate. We applied the dominant and recessive model in comparing clinical characteristics. Data are expressed in mean \pm SD for numerical data and $\mathrm{n}(\%)$ for categorical data. Allele and genotype frequencies were evaluated by Hardy-Weinberg equilibrium (HWE) using chi-square. The association between responder status and genotypes was analyzed using multinominal logistic regression by adjusting for age, gender, BMI, WC, lipid profiles, glomerular filtration rate, and blood pressure. All statistical analysis was performed using SPSS version 25.0 , and $p<0.05$ was considered statistically significant.

\section{Results}

Of the 191 participants who have classical T2DM signs, 62 were dropped out: 35 were $\mathrm{HbA} 1 \mathrm{c}<7 \%, 3$ were age $>70$ years old, $18 \mathrm{had}$ eGFR $<30 \mathrm{~mL} / \mathrm{min} / \mathrm{m} 2,20$ did not comply with consuming metformin, and 23 needed the second agent. The baseline characteristics are shown in Table. 1. BMI in the AA group $(29.00 \pm 5.34)$ was higher than the GG+AG group $(25.01 \pm 4.21)$ in the rs 2796498 recessive model $(p=0.04)$. However, both have been classified as obesity. The differences between FBP and $\mathrm{HbA} 1 \mathrm{c}$ before metformin treatment are only found in the rs 2796498 recessive model ( $p=0.02$ and $p=0.04$, respectively).

Specifically, rs 2796498 in the recessive model showed that the GG+GT group tended to have lower FBG and HbA1c before metformin therapy (174.39 \pm 58.08 $\mathrm{mg} / \mathrm{dL}$ and $9.29 \pm 1.74 \%)$ than the TT group $(211.67 \pm 74.21 \mathrm{mg} / \mathrm{dL}$ and $10.27 \pm 2.07 \%)$. Moreover, there were no significant differences in baseline characteristics in rs980377, either dominant or recessive models.

Table 1. Baseline characteristics in drug-naïve T2DM patients before receiving monotherapy metformin based on PRKAA2 genetic variation 


\begin{tabular}{|c|c|c|c|c|c|c|c|c|c|c|}
\hline \multirow[t]{3}{*}{ Characteristics } & \multicolumn{6}{|l|}{ rs2796498 } & \multicolumn{4}{|l|}{ rs9803799 } \\
\hline & \multicolumn{3}{|l|}{ Dominant } & \multicolumn{3}{|l|}{ Recessive } & \multicolumn{3}{|l|}{ Dominant } & \multirow{2}{*}{$\begin{array}{l}\text { Recess } \\
T T+G T\end{array}$} \\
\hline & GG & $A G+A A$ & $\begin{array}{l}\mathrm{p}- \\
\text { value }\end{array}$ & $G G+A G$ & $\mathrm{AA}$ & $\begin{array}{l}\mathrm{p}- \\
\text { value }\end{array}$ & TT & $\mathrm{GT}+\mathrm{GG}$ & $\begin{array}{l}\mathrm{p}- \\
\text { value }\end{array}$ & \\
\hline Age (years) & $52.73 \pm 9.00$ & $53.04 \pm 10.15$ & 0.87 & $9.52 \pm 0.95$ & $54.60 \pm 9.56$ & 0.68 & $52.92+9.18$ & $52.23 \pm 11.85$ & 0.80 & $53.02 \pm$ \\
\hline \multirow{2}{*}{$\begin{array}{l}\text { Sex } \\
\text { (male/female) }\end{array}$} & $20(69.0) /$ & $9(31.0) /$ & 0.09 & $28(96.6) /$ & $1(3.4) /$ & 0.71 & $25(86.2) /$ & $4(13.8) /$ & 0.77 & $28(96$. \\
\hline & $39(50.6)$ & $38(49.4)$ & & 73 (94.8) & $4(5.2)$ & & 68 (88.3) & 9 (11.7) & & $76(98$. \\
\hline BMI (kg/m2) & $24.64 \pm 4.11$ & $25.89 \pm 4.54$ & 0.14 & $25.01 \pm 4.21$ & $29.00 \pm 5.34$ & $0.04 *$ & $25.14 \pm 4.39$ & $25.62 \pm 4.01$ & 0.71 & $25.21 \pm$ \\
\hline WC (cm) & $86.92 \pm 7.56$ & $87.70 \pm 10.75$ & 0.67 & $86.91 \pm 9.07$ & $94.40 \pm 6.19$ & 0.07 & $87.12 \pm 9.40$ & $88.31 \pm 6.80$ & 0.66 & $87.30 \pm$ \\
\hline $\mathrm{SBP}(\mathrm{mmHg})$ & $129.92 \pm 20.54$ & $128.45 \pm 16.83$ & 0.69 & $128.93 \pm 18.97$ & $136.00 \pm 18.29$ & 0.42 & $129.42 \pm 17.99$ & $128.15 \pm 25.45$ & 0.82 & 129.37 \\
\hline $\mathrm{DBP}(\mathrm{mmHg})$ & $80.56 \pm 9.56$ & $79.38 \pm 8.09$ & 0.50 & $80.21 \pm 9.05$ & $76.60 \pm 5.03$ & 0.38 & $79.84 \pm 7.36$ & $81.46 \pm 16.65$ & 0.74 & $80.00 \pm$ \\
\hline $\mathrm{FBG}(\mathrm{mg} / \mathrm{dL})$ & $171.12 \pm 51.64$ & $192.77 \pm 72.38$ & 0.08 & $180.36 \pm 62.26$ & $188.00 \pm 70.76$ & 0.79 & $180.88 \pm 63.03$ & $179.54 \pm 59.43$ & 0.94 & 180.42 \\
\hline HbA1c (\%) & $9.21 \pm 1.67$ & $9.78 \pm 1.98$ & 0.11 & $9.44 \pm 1.81$ & $9.84 \pm 2.35$ & 0.63 & $9.49 \pm 1.84$ & $9.21 \pm 1.79$ & 0.60 & $9.47 \pm 1$ \\
\hline $\mathrm{CrSr}(\mathrm{mg} / \mathrm{dL})$ & $0.72 \pm 0.16$ & $0.71 \pm 0.22$ & 0.91 & $0.72 \pm 1.19$ & $0.66 \pm 0.12$ & 0.48 & $0.71 \pm 0.18$ & $0.73 \pm 0.21$ & 0.79 & $0.72 \pm 0$ \\
\hline $\begin{array}{l}\text { eGFR } \\
\left(\mathrm{mL} / \mathrm{menit} / \mathrm{m}^{2}\right)\end{array}$ & $99.34 \pm 16.15$ & $96.38 \pm 19.32$ & 0.40 & $98.09 \pm 17.73$ & $97.20 \pm 15.96$ & 0.91 & $98.37 \pm 17.09$ & $95.58 \pm 21.63$ & 0.61 & $97.78 \pm$ \\
\hline
\end{tabular}

Continuous data are presented in mean \pm SD and categorical data as $\mathrm{n}(\%)$

BMI: body mass index, WC: waist circumference, SBP: systolic blood pressure, DBP: diastolic blood pressure, FBG: fasting blood glucose, CrSr: creatinine serum, eGFR: estimated glomerular filtration rate, HDL-c: high density lipoprotein cholesterol, LDL-c: low density lipoprotein cholesterol, TC: total cholesterol

Allele and genotype frequency of PRKAA2 genetic variation in responders and non-responder of metformin therapy are listed in Table 2. The HWE of rs2796498, rs9803799, and rs 2746342 were $0.47,0.03$, and 0.92 , respectively. It suggested that the PRKAA2 genotypes of rs 2796498 and rs 2746342 in our population are consistent with HWE, but not rs9803799 ( $p<0.05$ ). Minor allele frequencies (MAFs) of rs2796498, rs9803799, and rs2746342 were $27.8 \%$ and $22.1 \%, 10.0 \%$ and $4.9 \%, 44,4 \%$ and $39.3 \%$, respectively, in responders and non-responders group. As presented in Table 2 ., the frequency of allele G, T, and G of rs2796498, rs9803799, and rs2746342, respectively, tend to higher in metformin non-responders than responders group. Nonetheless, we could not find the difference statistically of allele frequency between responders and non-responders ( $p=0.35$ for rs $2796498, p=0.15$ for $r s 9803799$, and $p=0.46$ for rs 2746342 ). We could not find any difference in blood pressure, FBG, HbA1c, HBA1c change, and lipid profiles after receiving metformin therapy based on $P R K A A 2 \mathrm{genetic}$ variation (Table. 3).

Table 2. Comparison of allelic frequency of PRKAA2 rs2796498, rs9803799, and rs2746342 variants distribution in responders and non-responder of metformin therapy 


\begin{tabular}{|c|c|c|c|c|}
\hline SNP ID & Genotype & $\begin{array}{l}\text { Responders } \\
(n=45)\end{array}$ & $\begin{array}{l}\text { Non responders } \\
(n=61)\end{array}$ & $\mathrm{p}$-value \\
\hline \multirow[t]{7}{*}{ rs2796498 } & GG & $22(48.9)$ & $37(60.7)$ & 0.44 \\
\hline & $A G$ & $21(46.7)$ & $21(34.4)$ & \\
\hline & AA & $2(4.4)$ & $3(4.9)$ & \\
\hline & Alleles & & & \\
\hline & G & 65 (72.2) & 95 (77.9) & 0.35 \\
\hline & A & $25(27.8)$ & $27(22.1)$ & \\
\hline & HWE-p value & 0.47 & & \\
\hline \multirow[t]{7}{*}{ rs9803799 } & TT & 37 (82.2) & $56(91.8)$ & 0.31 \\
\hline & GT & 7 (15.6) & $4(6.6)$ & \\
\hline & GG & $1(2.2)$ & $1(1.6)$ & \\
\hline & Alleles & & & \\
\hline & $\mathrm{T}$ & $81(90.0)$ & $116(95.1)$ & 0.15 \\
\hline & G & $9(10.0)$ & $6(4.9)$ & \\
\hline & HWE-p value & 0.03 & & \\
\hline \multirow[t]{7}{*}{ rs2746342 } & GG & $12(26.7)$ & 24 (39.3) & 0.28 \\
\hline & $\mathrm{GT}$ & $26(57.8)$ & $26(42.6)$ & \\
\hline & TT & 7 (15.5) & $11(18.1)$ & \\
\hline & Alleles & & & \\
\hline & G & $50(55.6)$ & $74(60.7)$ & 0.46 \\
\hline & $\mathrm{T}$ & $40(44.4)$ & 48 (39.3) & \\
\hline & HWE-p value & 0.92 & & \\
\hline
\end{tabular}

Table 3. Follow-up characteristics in drug-naïve T2DM patients after receiving monotherapy metformin based on PRKAA2 genetic variation

\begin{tabular}{|c|c|c|c|c|c|c|c|c|c|c|}
\hline \multirow[t]{3}{*}{ Characteristics } & \multicolumn{6}{|l|}{ rs2796498 } & \multicolumn{4}{|l|}{ rs9803799 } \\
\hline & \multicolumn{3}{|l|}{ Dominant } & \multicolumn{3}{|l|}{ Recessive } & \multicolumn{3}{|l|}{ Dominant } & \multirow{2}{*}{$\begin{array}{l}\text { Recessi } \\
T T+G T\end{array}$} \\
\hline & GG & $A G+A A$ & $\begin{array}{l}\mathrm{p}- \\
\text { value }\end{array}$ & $G G+A G$ & AA & $\begin{array}{l}\mathrm{p}- \\
\text { value }\end{array}$ & TT & $\mathrm{GT}+\mathrm{GG}$ & $\begin{array}{l}\mathrm{p}- \\
\text { value }\end{array}$ & \\
\hline $\mathrm{SBP}(\mathrm{mmHg})$ & $126.03 \pm 12.63$ & $124.79 \pm 7.89$ & 0.56 & $125.36 \pm 10.94$ & $128.00 \pm 6.16$ & 0.59 & $125.77 \pm 11.14$ & $123.38 \pm 7.52$ & 0.46 & 125.52 \\
\hline $\mathrm{DBP}(\mathrm{mmHg})$ & $80.69+6.94$ & $80.43+6.57$ & 0.84 & $80.59 \pm 6.83$ & $80.20 \pm 5.54$ & 0.90 & $80.48+6.91$ & $81.23 \pm 5.67$ & 0.71 & $80.63 \pm t$ \\
\hline $\begin{array}{l}\text { FBG-post } \\
\text { (mg/dL) }\end{array}$ & $134.66 \pm 29.85$ & $139.53 \pm 28.48$ & 0.40 & $135.91 \pm 28.72$ & $155.20 \pm 36.63$ & 0.15 & $138.38 \pm 29.66$ & $125.69 \pm 23.88$ & 0.14 & 137.09 \\
\hline HbA1c(\%) & $8.16 \pm 1.26$ & $8.42 \pm 1.33$ & 0.31 & $8.25 \pm 1.29$ & $8.84 \pm 1.27$ & 0.32 & $8.35 \pm 1.32$ & $7.73 \pm 0.94$ & 0.11 & $8.29 \pm 1$. \\
\hline $\begin{array}{l}\text { HbA1c } \\
\text { changes (\%) }\end{array}$ & $1.03 \pm 1.18$ & $1.36 \pm 1.76$ & 0.25 & $1.19 \pm 1.48$ & $1.00 \pm 1.20$ & 0.78 & $1.14 \pm 1.51$ & $1.48 \pm 1.11$ & 0.43 & $1.17 \pm 1$ \\
\hline $\begin{array}{l}\mathrm{HDL}-\mathrm{c} \\
(\mathrm{mg} / \mathrm{dL})\end{array}$ & $47.90 \pm 8.40$ & $47.45 \pm 8.34$ & 0.78 & $47.80 \pm 8.39$ & $45.60 \pm 7.57$ & 0.57 & $47.51 \pm 8.62$ & $49.08 \pm 6.02$ & 0.53 & $47.56+\varepsilon$ \\
\hline LDL-c (mg/dL) & $104.02 \pm 24.89$ & $110.43 \pm 32.71$ & 0.26 & $106.16 \pm 28.70$ & $121.00 \pm 26.66$ & 0.26 & $108.56 \pm 28.44$ & $94.69 \pm 28.31$ & 0.10 & 106.67껌 \\
\hline TC (mg/dL) & $177.25 \pm 36.12$ & $183.98 \pm 34.01$ & 0.33 & $179.46 \pm 35.36$ & $196.00 \pm 30.49$ & 0.31 & $181.81 \pm 35.43$ & $169.00 \pm 32.55$ & 0.22 & 179.77포 \\
\hline $\begin{array}{l}\text { Triglycerides } \\
(\mathrm{mg} / \mathrm{dL})\end{array}$ & $140.98 \pm 61.60$ & $130.45 \pm 51.07$ & 0.35 & $135.74 \pm 35.77$ & $147.80 \pm 46.18$ & 0.65 & $137.69 \pm 58.45$ & $126.46 \pm 47.14$ & 0.51 & 135.76 \\
\hline
\end{tabular}

Data are presented in mean $\underline{\mathbf{S D}}$

BMI: body mass index, WC: waist circumference, SBP: systolic blood pressure, DBP: diastolic blood pressure, FBG: fasting blood glucose, CrSr: creatinine serum, eGFR: estimated glomerular filtration rate, HDL-c: high density lipoprotein cholesterol, LDL-c: low density lipoprotein cholesterol, TC: total cholesterol 
Furthermore, multinominal logistic regression models were applied to verify the effect of PRKAA2 genetic variation on metformin efficacy (Table 4). This study failed to detect the association between PRKAA2 genetic variation with metformin efficacy, even after adjusting age, gender, and adjusting for BMI, WC, and lipid profiles. Interestingly, we found some association after adjusting for BMI, WC, lipid profiles, blood pressure, and eGFR. As shown in table 4. AG of the rs2796498 genetic variation was statistically significant to decrease metformin efficacy (OR=0.32, 95\%Cl=0.12 - 0.86). Dominant model of rs 2796498 (GG vs. $A G+A A)$ showed significant association to metformin efficacy $(O R=0.34,95 \% \mathrm{Cl}=0.13-0.90)$.

Table 4. Association of PRKAA2 genetic variations between responders and non-responders receiving metformin therapy

\begin{tabular}{|c|c|c|c|}
\hline Genotype & OR $(95 \% \mathrm{Cl})$ & $\operatorname{AOR}(95 \% \mathrm{Cl})^{\mathrm{a}}$ & $\operatorname{AOR}(95 \% \mathrm{Cl})^{\mathrm{b}}$ \\
\hline \multicolumn{4}{|l|}{ rs2796498 } \\
\hline GG & 1.00 (reference) & & \\
\hline AG & $0.57(0.25-1.27)$ & $0.61(0.27-1.37)$ & $0.32(0.12-0.86)^{\star}$ \\
\hline AA & $0.85(0.13-5.51)$ & $0.90(0.14-5.85)$ & $0.67(0.07-6.50)$ \\
\hline Dominant (GG vs. AG+AA) & $0.60(0.27-1.30)$ & $0.63(0.29-1.40)$ & $0.34(0.13-0.90) *$ \\
\hline Recessive (GG+AG vs. AA) & $1.09(0.17-6.79)$ & $1.12(0.92-1.13)$ & $1.15(0.13-10.46)$ \\
\hline G allele & 1.00 (reference) & & \\
\hline A allele & $0.75(0.40-1.42)$ & $0.75(0.40-1.41)$ & $0.55(0.26-1.14)$ \\
\hline \multicolumn{4}{|l|}{ rs9803799 } \\
\hline TT & 1.00 (reference) & & \\
\hline GT & $0.37(0.10-1.35)$ & $0.37(0.10-1.36)$ & $0.45(0.09-2.16)$ \\
\hline GG & $0.65(0.04-10.61)$ & $0.95(0.04-20.15)$ & $0.14(0.00-54.13)$ \\
\hline Dominant (TT vs. GT+GG) & $0.40(0.12-1.33)$ & $0.41(0.12-1.36)$ & $0.45(0.11-1.86)$ \\
\hline Recessive (TT+GT vs. GG) & $0.72(0.04-11.77)$ & $1.05(0.05-22.20)$ & $0.14(0.00-54.62)$ \\
\hline T allele & 1.00 (reference) & & \\
\hline G allele & $0.46(0.16-1.34)$ & $0.46(0.16-1.37)$ & $0.52(0.15-1.77)$ \\
\hline \multicolumn{4}{|l|}{ rs2746342 } \\
\hline GG & 1.00 (reference) & & \\
\hline GT & $0.46(0.19-1.12)$ & $0.52(0.21-1.26)$ & $0.43(0.16-1.19)$ \\
\hline TT & $0.72(0.22-2.36)$ & $0.83(0.25-2.71)$ & $0.65(0.17-2.50)$ \\
\hline Dominant (GG vs. GT+TT) & $0.51(0.22-1.21)$ & $0.58(0.25-1.35)$ & $0.47(0.18-1.24)$ \\
\hline Recessive (GG+GT vs. TT) & $1.16(0.41-3.29)$ & $1.24(0.43-3.53)$ & $1.08(0.33-3.55)$ \\
\hline G allele & 1.00 (reference) & & \\
\hline T allele & $0.81(0.47-1.41)$ & $0.83(0.48-1.45)$ & $0.78(0.42-1.44)$ \\
\hline
\end{tabular}

${ }^{*} p<0.05$

a adjusted for age and gender, ${ }^{b}$ adjusted for BMI, WC, lipid profiles, glomerular filtration rate, and blood pressure.

\section{Discussion}

The previous study found an association between rs2796498 and rs2746342 with T2DM susceptibility [21, 22], and rs9803799 with metformin efficacy [23]. Accordingly, this current study evaluates the impact of PRKAA2 genetic variation on metformin efficacy. To the authors knowledge, this study is the first pharmacogenomic research reporting allele frequency of rs2796498, rs9803799, and rs2746342 applied metformin therapy among Indonesia drug-naïve T2DM patients.

This recent study found that T2DM patients with AA genotype of rs2796498 had significantly higher BMI than GG+AG. However, this significant mean of BMI difference was not observed in rs9803799 and rs2746342. These findings might imply that the AA genotype of rs 2796498 is associated with obesity, then it should be correlated with lipid profiles. Our findings contradict the data obtained by Jones et al. [25], which indicated that rs 2796498 and rs2746342 genetic variations correlated lipid profiles. Furthermore, this study found that the TT genotype of rs 2746342 had higher FBG and HbA1c levels significantly than GG+GT among study subjects before receiving metformin. Conversely, Shen et al. reported that the G allele had a higher FBG level and T2DM risk [22] . It could 
be caused by dissimilar ethnicity, where Shen et al. focused on Han Chinese, and this study focused on Indonesia. Nevertheless, the recessive model of rs2746342 did not influence the difference in FBG, $\mathrm{HbA1c}$, and $\mathrm{HbA} 1 \mathrm{c}$ change after metformin therapy. It could be an early indicator that rs2746342 genetic variation does not alter metformin efficacy in our population.

AMPK, as the main target of metformin, regulates the function of hepatic glucose metabolism and pancreatic b-cell $[8,11,26]$. It has been widely agreed that the phosphorylation of AMPK is induced by metformin, although the specific route is not clear yet. An animal study confirmed that 10 weeks of metformin treatment significantly escalated AMPK phosphorylation on the a2 subunit [27]. Several PRKAA2 genetic variations have been investigated to increase T2DM risk, including that's located in the intron region [21, 22, 28]. Nevertheless, only a few studies observe that SNP is related to metformin responses [23]. A review has been mentioned that PRKAA2 genetic variation is one of the genes contributing to the metformin mechanism [20]. However, it has not yet been discovered clearly in a clinical study.

Thus, this study investigated the association of PRKAA2 genetic variation and metformin efficacy among drug-naïve T2DM patients. Our results discovered that allele and genotype frequencies of rs2796498, rs9803799, and rs2746342 between responders and non-responders were not significantly different. Interestingly, the wild type of rs2796498 (AA) and rs9803799 (GG) are detected in little number, either in the responder or non-responder group. It confirmed that those SNP has related to T2DM [21, 29], and our study participants were T2DM, indeed.

Moreover, we found a significant association between rs2796498 in AG genotype and dominant model with metformin efficacy, after adjusting for BMI, WC, lipid profiles, blood pressure, and eGFR. AG carrier in rs2796498 had 0.32 times of metformin response compared with the GG carrier. Therefore, T2DM patients with $A G$ carriers might have a poor response to metformin therapy. Apart from these, the dominant model found that $A G+A A$ had a worse metformin efficacy compared with GG in drug-naïve T2DM patients. However, we could not detect that the A allele influences metformin efficacy. This point emphasizes that AG carrier in rs 2796498 is the predictive factors of metformin efficacy in our population. Although metformin is well-known as a better treatment for T2DM patients with obesity (ESC/EASD) [2], a different BMI in baseline could influence the impact of AA genotype in metformin efficacy.

On the other hand, we could not identify any relationship between rs 9803799 and metformin efficacy. Conversely, a study in the U.S population reported that rs9803799 had significant interaction with metformin [23]. In addition, rs9803799 deviated from HWE. Therefore, findings related to rs980799 may be reported bias association [30].

This study still has several limitations. First, the epistatic mechanism may contribute to metformin efficacy. It is possible if other genes or SNPs also affect metformin response. Second, environmental factors such as diet, physical activities, and adherence might impact decreasing HbA1c. Third, an SNP was not in agreement with HWE. Finally, the sample size should be larger to detect the function of PRKAA2 in metformin efficacy. Further replication pharmacogenomic studies are needed to confirm the PRKAA2 genetic variation on metformin efficacy.

\section{Conclusions}

In summary, there were no significant differences between genotype and allele frequencies of PRKAA2 genetic variation with metformin efficacy. Only AG genotype and dominant model of PRKAA2 rs2796498 associated with metformin efficacy in drug-naïve T2DM patients treated with metformin as monotherapy, after adjusting for BMI, WC, blood pressure, eGFR, and lipid profiles. Nonetheless, PRKAA2 rs9803799 and rs 2746342 might have no impact on metformin efficacy among Indonesian. Further study recruiting a larger sample size and engaging environment factors such as physical activities are required to confirm the role of PRKAA2 genetic variation, especially of rs2796498, rs9803799, and rs274634 on metformin efficacy among drug-naïve T2DM patients.

\section{Abbreviation}

T2DM: type 2 diabetes mellitus

AMPK: AMP-activated protein kinase

ACC: acetyl-CoA carboxylase

PHC: primary health care

FBG: fasting blood glucose

BMI: body mass index

WC: waist circumference

SBP: systolic blood pressure

DBP: diastolic blood pressure

FBG: fasting blood glucose

CrSr: creatinine serum

eGFR: estimated glomerular filtration rate 
HDL-c: high density lipoprotein cholesterol

LDL-c: low density lipoprotein cholesterol

TC: total cholesterol

\section{Declarations}

\section{ACKNOWLEDGEMENTS}

We are grateful to all the participants for their cooperation and the involved health practitioners in PHC, Sleman Districts. The authors thank Prof. Iwan Dwiprahasto, M.Med.Sc., Ph.D for supporting the study.

\section{ETHICS APPROVAL AND CONSENT TO PARTICIPATE}

The study protocol was approved by the Medical and Health Research Ethics Committee (MHREC) Faculty of Medicine, Public Health, and Nursing Universitas Gadjah Mada - Dr. Sardjito General Hospital. All measurements and data collected were done after obtaining informed consent signed by all participants.

\section{CONSENT FOR PUBLICATION}

Not applicable.

\section{AVAIBILITY OF DATA AND MATERIALS}

All data generated or analysed during this study are included in this published article (Additional file 1).

\section{COMPETING INTERESTS}

There are no conflicts of interest to declare.

\section{FUNDING}

This study was funded by Indonesia Endowment Fund for Education (LPDP) and Rekognisi Tugas Akhir grant, Universitas Gadjah Mada.

\section{AUTHOR'S CONTRIBUTION}

DV: collected patient's data in primary health care, conducted the genetic analysis, analyzed data, and wrote the manuscript. MW: designed and managed the study, wrote and corrected the manuscript. DN: interpreted the results and wrote the manuscript. All authors read and approved the final manuscript.

AUTHOR INFORMATION

Affiliation

Department of Pharmacology and Therapy, Faculty of Medicine, Public Health, and Nursing, Universitas Gadjah Mada, Yogyakarta, Indonesia-55281

Dita Maria Virginia, Mae Sri Hartati Wahyuningsih, Dwi Aris Agung Nugrahaningsih

Faculty of Pharmacy, Universitas Sanata Dharma, Yogyakarta, Indonesia-55281

Dita Maria Virginia

Center of Genetic Study, Faculty of Medicine, Public Health, and Nursing, Universitas Gadjah Mada, Yogyakarta, Indonesia-55281

Dwi Aris Agung Nugrahaningsih

Corresponding author

Dwi Aris Agung Nugrahaningsih

\section{References}

[1] American Diabetes Association. Standards of Medical Care in Diabetes-2018. Diabetes Care 2018; 41: 14-37.

[2] Cosentino F, Grant PJ, Aboyans V, et al. 2019 ESC Guidelines on diabetes, pre-diabetes, and cardiovascular diseases developed in collaboration with the EASD. Eur Heart J 2020; 41: 255-323.

[3] Schlender L, Martinez Y V., Adeniji C, et al. Efficacy and safety of metformin in the management of type 2 diabetes mellitus in older adults: A systematic review for the development of recommendations to reduce potentially inappropriate prescribing. BMC Geriatrics; 17 . Epub ahead of print October $16,2017$. DOI: 10.1186/s12877-017-0574-5. 
[4] Gong L, Goswami S, Giacomini KM, et al. Metformin pathways. Pharmacogenet Genomics 2012; 22: 820-827.

[5] Kinaan M, Ding H, Triggle CR. Metformin: An Old Drug for the Treatment of Diabetes but a New Drug for the Protection of the Endothelium. Medical Principles and Practice 2015; 24: 401-415.

[6] Yan Y, L. Kover K, V. Moore W. New Insight into Metformin Mechanism of Action and Clinical Application. In: Metformin [Working Title]. IntechOpen. Epub ahead of print March 20, 2020. DOI: 10.5772/intechopen.91148.

[7] Rena G, Hardie DG, Pearson ER. The mechanisms of action of metformin. Diabetologia 2017; 60: 1577-1585.

[8] Wang Y, An H, Liu T, et al. Metformin Improves Mitochondrial Respiratory Activity through Activation of AMPK. Cell Rep 2019; 29: 1511-1523.e5.

[9] Garcia D, Shaw RJ. AMPK: mechanisms of cellular energy sensing and restoration of metabolic balance Daniel. Mol Cell 2017; 66: 789-800.

[10] Meng S, Cao J, He Q, et al. Metformin activates AMP-activated protein kinase by promoting formation of the aßyheterotrimeric complex. J Biol Chem 2015; 290: 3393-3802.

[11] Agius L, Ford BE, Chachra SS. The metformin mechanism on gluconeogenesis and AMPK activation: The metabolite perspective. Int J Mol Sci; 21. Epub ahead of print 2020. DOI: 10.3390/ijms21093240.

[12] Srivastava RAK, Pinkosky SL, Filippov S, et al. AMP-activated protein kinase: An emerging drug target to regulate imbalances in lipid and carbohydrate metabolism to treat cardio-metabolic diseases. Journal of Lipid Research 2012; 53: 2490-2514.

[13] Fullerton MD, Galic S, Marcinko K, et al. Single phosphorylation sites in Acc1 and Acc2 regulate lipid homeostasis and the insulin-sensitizing effects of metformin. Nat Med 2013; 19: 1649-1654.

[14] Rashid M, Shahzad M, Mahmood S, et al. Variability in the therapeutic response of metformin treatment in patients with type 2 diabetes mellitus. Pakistan J Med Sci 2019; 35: 71-76.

[15] Zhang J, Wang N, Xing X, et al. Factors that influence the efficacy of acarbose and metformin as initial therapy in Chinese patients with newly diagnosed type 2 diabetes: A subanalysis of the MARCH trial. Curr Med Res Opin 2016; 32: 713-719.

[16] Ko S-H, Rhee SY, Kim HJ, et al. Monotherapy in patients with type 2 diabetes mellitus. Korean J Intern Med Korean J Intern Med 2005; 32: $959-966$.

[17] Franks PW, Pearson E, Florez JC. Gene-environment and gene-treatment interactions in type 2 diabetes: Progress, pitfalls, and prospects. Diabetes Care 2013; 36: 1413-1421.

[18] Yoon H, Cho H-Y, Yoo H-D, et al. Influences of Organic Cation Transporter Polymorphisms on the Population Pharmacokinetics of Metformin in Healthy Subjects. AAPS J 2013; 15: 571-580.

[19] Malodobra-Mazur M, Bednarska-Chabowska D, Olewinski R, et al. Single nucleotide polymorphisms in 5'-UTR of the SLC2A4 gene regulate solute carrier family 2 member 4 gene expression in visceral adipose tissue. Gene 2016; 576: 499-504.

[20] Dawed AY, Zhou K, Pearson ER. Pharmacogenetics in type 2 diabetes: influence on response to oral hypoglycemic agents. Pharmgenomics Pers Med 2016; 9: 17-29.

[21] Li Q, Li C, Li H, et al. Effect of AMP-activated protein kinase subunit alpha 2 (PRKAA2) genetic polymorphisms on susceptibility to type 2 diabetes mellitus and diabetic nephropathy in a Chinese population: PRKAA2. J Diabetes 2018; 10: 43-49.

[22] Shen J-Z, Ge W-H, Fang Y, et al. A novel polymorphism in protein kinase AMP-activated catalytic subunit alpha 2 ( PRKAA2) is associated with type 2 diabetes in the Han Chinese population. J Diabetes 2017; 9: 606-612.

[23] Jablonski KA, McAteer JB, de Bakker PIW, et al. Common Variants in 40 Genes Assessed for Diabetes Incidence and Response to Metformin and Lifestyle Intervention in the Diabetes Prevention Program. Diabetes 2010; 59: 2672-2681.

[24] Hirst JA, Farmer AJ, Ali R, et al. Quantifying the Effect of Metformin Treatment and Dose on Glycemic Control. Diabetes Care; 35. Epub ahead of print 2012. DOI: $10.2337 / \mathrm{dc} 11-1465$.

[25] Spencer-Jones NJ, Ge D, Snieder H, et al. AMP-kinase alpha2 subunit gene PRKAA2 variants are associated with total cholesterol, low-density lipoproteincholesterol and high-density lipoprotein-cholesterol in normal women. J Med Genet 2006; 43: 936-42.

[26] Szkudelski T, Szkudelska K. The relevance of AMP-activated protein kinase in insulin-secreting $\beta$ cells: a potential target for improving $\beta$ cell function? Journal of Physiology and Biochemistry 2019; 75: 423-432.

[27] Musi N, Hirshman MF, Nygren J, et al. Metformin Increases AMP-Activated Protein Kinase Activity in Skeletal Muscle of Subjects With Type 2 Diabetes. Diabetes 2002; 51: 2074-2081. 
[28] Sun MW, Lee JY, De Bakker PIW, et al. Haplotype structures and large-scale association testing of the 5' AMP-activated protein kinase genes PRKAA2, PRKAB1, and PRKAB1 with type 2 diabetes. Diabetes 2006; 55: 849-855.

[29] Jablonski KA, McAteer JB, De Bakker PIW, et al. Common variants in 40 genes assessed for diabetes incidence and response to metformin and lifestyle intervention in the diabetes prevention program. Diabetes 2010; 59: 2672-2681.

[30] Namipashaki A, Razaghi-Moghadam Z, Ansari-Pour N. The essentiality of reporting Hardy-Weinberg equilibrium calculations in population-based genetic association studies. Cell Journal 2015; 17: 187-192.

\section{Supplementary Files}

This is a list of supplementary files associated with this preprint. Click to download.

- BMCAdditionalFile1PRKAA2metformin.xIsx

- BMCAdditionalFile1PRKAA2metformin.xIsx 